\title{
BMJ Open Enablers and barriers to improving worksite canteen nutrition in Pudong, China: a mixed-methods formative research study
}

\author{
Ruoran Li, ${ }^{1}$ You Wu, ${ }^{2}$ Limei Jing, ${ }^{3}$ Lindsay M Jaacks ${ }^{4}$
}

To cite: Li R, Wu Y, Jing L, et al. Enablers and barriers to improving worksite canteen nutrition in Pudong, China: a mixed-methods formative research study. BMJ Open 2018:8:e020529. doi:10.1136/ bmjopen-2017-020529

- Prepublication history and additional material for this paper are available online. To view these files, please visit the journal online (http://dx.doi. org/10.1136/bmjopen-2017020529).

$\mathrm{LJ}$ and $\mathrm{LMJ}$ are $\mathrm{Co}$-senior authors.

Received 8 November 2017 Revised 9 March 2018 Accepted 13 March 2018

\section{Check for updates}

${ }^{1}$ Department of Epidemiology, Harvard T.H. Chan School of Public Health, Boston, Massachusetts, USA ${ }^{2}$ Department of Nutrition, Harvard T.H. Chan School of Public Health, Boston, Massachusetts, USA ${ }^{3}$ Pudong Institute for Health Development, Shanghai, China ${ }^{4}$ Department of Global Health and Population, Harvard T.H. Chan School of Public Health, Boston, Massachusetts, USA

Correspondence to Dr Lindsay M Jaacks; jaacks@hsph.harvard.edu

\section{ABSTRACT}

Objective To identify individual-level and organisationlevel enablers and barriers to the provision and consumption of healthier foods at worksite canteens in China and to develop a theoretical framework and evidence-based, specific, practical intervention strategies. Design Mixed-methods formative research, with in-depth interviews, focus group discussions and quantitative questionnaires.

Setting Two community health centres (CHCs) in Pudong, Shanghai, China.

Participants In-depth interviews with three $\mathrm{CHC}$ administrators and three canteen managers and staff. Six focus groups with a total of 19 male and 36 female employees, aged 25-67 years.

Results Three subthemes were identified as important for influencing individual food choice: the cultural perception of 'eating well', the need to balance taste preferences and nutrition, and the emphasis on food safety in healthfulness. At the organisation level, two related subthemes emerged: the balance of canteen budget and food safety with the variety and quality of offerings, and the interplay between key stakeholders. Key barriers included cost, poor communication between employees and management, individuals' emphasis on taste over healthfulness, variation in individual preferences and discordance between perceived and actual weight status, particularly among men. Key enablers included strong, positive food culture in China and trust in canteen food. An ecological framework to describe determinants of worksite food environment in Shanghai was developed and intervention strategies were mapped onto this framework.

Conclusions $A$ balancing act occurs at multiple levels and ultimately determines the worksite food environment and employee food choice at CHCs in Shanghai of China. There is a need to implement these findings and evaluate their impact on diet and health.

\section{INTRODUCTION}

China's recent nutrition transition has resulted in significant increases in the prevalence of cardiometabolic diseases including diabetes and hypertension over the past three decades. ${ }^{1}$ Rapid urbanisation accelerated this transition. With over $20 \%$ more population

\section{Strengths and limitations of this study}

- Our study is the first to describe determinants of the worksite food environment and employee food choice in China, and informs context-specific, culturally appropriate interventions in this setting.

- The willingness to discuss food in the Chinese culture promoted a high level of engagement of employees (eg, healthcare providers), administrators and canteen staff at sampled community health centres.

- The interviewer, a native speaker with an understanding of local culture, coded the transcripts and analysed emerging themes, which ensured minimal discrepancy between field observations and subsequent analyses.

- At the time of the site visits, there were few opportunities to directly observe usual canteen food behaviours because of construction and restructuring at sampled community health centres.

- Employees who volunteered to be in the focus groups may be more interested in healthy food and therefore more supportive of making changes to the canteen-this potential source of bias should be considered when translating findings into worksite interventions.

living in urbanised areas in 2014 than in 1990, dietary patterns are increasingly shifting to high-fat, high-sugar and energy-dense diets. ${ }^{2}$

The translation of successful lifestyle interventions to real-world settings has been challenging. Worksites provide a unique opportunity for translating lifestyle interventions because employees spend most of their waking hours at worksites. In China, $91 \%$ of men and $74 \%$ of women aged $25-64$ years participate in the workforce, and $46 \%$ of workforce participants are employed in urban areas. ${ }^{3}$ A recent systematic review found that more than half $(13 / 22)$ of the worksite-based nutrition interventions evaluated reported significant improvements in eating behaviour including increased sales 
of healthy foods, increased fruit and vegetable consumption, and decreased calories purchased. ${ }^{4}$ To our knowledge, no study has evaluated a worksite-based nutrition intervention in China.

Re-established in 2006 to cover all residents in China, community health centres (CHCs) act as the universal primary healthcare system, integrating prevention, medical treatment, rehabilitation, health education and family planning services. ${ }^{5}$ Since the healthcare system reform in 2009, the number of CHCs in China increased by $26 \% .^{5}$ At the end of 2016, there were a total of 34327 CHCs with $>522000$ workers. ${ }^{6}$ In addition to forming a substantial proportion of the workforce, primary healthcare workers also serve as role models for the general public. $^{7}$ Thus, implementing worksite-based nutrition interventions for primary healthcare workers could lead to widespread public health impact in China.

The goal of this mixed-methods formative research study was to provide necessary information to inform the development of a canteen-based nutrition intervention to improve dietary intake and reduce cardiometabolic disease risk among employees of CHCs in China. The objectives were to (1) identify individual-level and organisation-level enablers and barriers to the provision and consumption of healthier foods at worksite canteens in China and (2) to develop a theoretical framework based on these findings and evidence-based, specific, practical intervention strategies.

\section{METHODS}

\section{Study setting}

We sampled two CHCs in Pudong, Shanghai (sites A and B), each employing approximately 150 employees. A population of 5.5 million resides in Pudong, contributing to $32 \%$ of Shanghai's overall GDP. In total, there are 46 CHCs in Pudong, offering 3188 in-patient beds and employing 6793 staff and healthcare personnel.

Site A serves an urban area with $>300000$ permanent residents. At the time of the site visit in June 2017, the CHC canteen was under renovation. The canteen food service was outsourced to a staffing management company, which offers a single lunch option for employees and posts menus twice weekly for subsequent days. Employees can sign up for lunch in the morning of each day, allowing the canteen staff to determine the amount of food that needs to be prepared. In addition to paying for canteen staffing, utilities and additional costs of purchasing cooking oil derived from non-genetically modified (GMO) soy, the CHC management pays for each portion of lunch at 7.5 RMB for the raw materials.

Site B operates on two campuses and serves a remote rural area with $<100000$ permanent residents. The canteen food service was also outsourced to a staffing management company (a different one than site A). For lunch, employees may choose one meat dish (out of two offered), one half-meat dish (out of two offered) and two vegetable dishes (out of three or four offered), with a portion of rice and free soup. Just prior to our site B visit, the CHC management closed the main campus kitchen indefinitely due to safety concerns, with no foreseeable funding for renovation. All canteen staff were relocated to the secondary campus. Each workday, lunch is prepared at and delivered from the secondary campus to the main campus. For each lunch portion, an employee pays 2.5 RMB out of pocket (through prepurchased vouchers), and the CHC management reimburses 2.5 RMB for the raw materials. The $\mathrm{CHC}$ management pays for canteen staffing and utilities.

\section{Sample population}

In-depth interviews were conducted with canteen managers (from the staffing management company) and CHC administrators: at site A, one canteen manager and one CHC administrator; at site $\mathrm{B}$, two canteen staff were interviewed together, and two $\mathrm{CHC}$ administrators were interviewed individually. Three focus groups were conducted at each site, with a total of 19 men and 36 women. Focus group participants were recruited by CHC administrators to represent frequent canteen users.

\section{Data collection}

Written informed consent was obtained from all participants.

The Pudong Institute for Health Development (PIHD) (LJ) had established relationships with all Pudong CHCs. LJ identified two CHCs for this study (one rural and one urban) and arranged site visits. Careful consideration was given to the composition of the focus groups to ensure that the environment was conducive to open discussions about diet and health. We purposively separated males and females, and physicians and nurses, considering Chinese cultural norms relating to diet and bodyweight, and power dynamics between medical personnel.

Prior to conducting the face-to-face interviews and focus groups, a trained interviewer (RL, a female doctoral student) discussed the consent form and a corresponding information sheet, and acquired the informed consent. The consent form disclosed the study's primary aim. All focus group participants were asked to complete a brief questionnaire that included standard questions on socio-demographics and self-reported height and weight. Body mass index (BMI) was calculated as weight in kilograms divided by height in metres-squared. In accordance with the Chinese government guideline, overweight was defined as BMI $\geq 24 \mathrm{~kg} / \mathrm{m}^{2}{ }^{8}$ Following interview guides (see online supplementary files 1 and 2 for English translations), RL conducted the in-depth interviews and focus groups in a designated private space within the CHC in the participants' native language (Mandarin). Each interview and focus group lasted approximately $60 \mathrm{~min}$. All participants agreed to participate and to be audio-recorded. RL made field notes. One researcher from PIHD was present in two focus groups and two interviews as an observer. 


\section{Data analysis}

Interviews and focus groups were transcribed and translated into English, and entered into Dedoose V.7.0.23 (SocioCultural Research Consultants, Los Angeles, California, USA) for analysis. RL and LMJ reviewed emergent codes after the transcription of one interview and one focus group, to ensure data quality and to allow for any necessary adjustments to the interview guides. After this first meeting and development of an initial codebook, RL separately coded subsequent transcripts in batches of 2-3 each, followed by additional meetings with LMJ and adjustment of the interview guides and/or codebook (see online supplementary file 3 ) as needed. Once all transcripts had been coded by RL and reviewed by LMJ, coding reports were exported from Dedoose and analysed using a constant comparative method by RL with input from LMJ. Original categories arising from the interviews and focus groups were applied to the cumulative data and revised multiple times until there was consensus about their fit and the emergent themes. Statistical tests of participant characteristics were conducted using Stata V.15.1 (StataCorp). We used Fisher's exact test for comparing categorical variables, and two-sample t-test with unequal variances for continuous variables.

\section{Patient and public involvement}

Patients and public were not involved in this study.

\section{RESULTS}

Three CHC administrators and two canteen managers were interviewed. Among the $\mathrm{CHC}$ administrators, we interviewed one female administrator who oversees the canteen at site $\mathrm{A}$, one male administrator who oversees the canteen at site B and the CHC director at site B (woman). Both canteen managers were women, each employed by the respective outsourced canteen company of each site.
Three single-sex focus groups were conducted at each site (table 1). Mean (SD) age ranged from 34.3 (6.1) years among the male focus group at site A to 44.4 (10.9) years among the male focus group at site $\mathrm{B}$. Male participants had higher BMI than females $(\mathrm{P}<0.001)$ : over half of men were overweight compared with $\leq 25 \%$ of women $(\mathrm{P}<0.001)$. Participants at site $\mathrm{B}$ (the rural site) had higher BMI $(\mathrm{P}=0.06)$ and were more likely to be overweight than participants at site A $(\mathrm{P}=0.08)$. Participants at site A were more likely to eat at the canteen regularly ( $\geq 3$ time/week) than participants at site $\mathrm{B}(\mathrm{P}=0.004)$.

We identified two primary themes and five subthemes, discussed in detail in the sections that follow.

\section{Theme 1: balancing acts that influence individual food choice} Subtheme 1a: cultural perception of 'eating well'

To 'eat well' was perceived by many employees as a 'basic right and key to happiness' (focus group, site $\mathrm{B}$, male physicians). This stemmed from the near universal belief among participants that traditional Chinese cuisine is good; in terms of taste, food presentation and variety in cooking methods and dishes prepared for each meal. For example, one CHC administrator at site B said:'We seldom make dishes with only one food [item]. We always pair the main food [item] with other ingredients. The cost of labor, raw food material, and time are not comparable to the Western's ... In Western countries, salad is a popular dish, a healthy dish, and an easy-to-make dish ... meanwhile it costs little. However, in China, we have so many cooking methods: fry, stir-fry, deep-fry, steam, et cetera.' This perception of inherent cooking abilities could prove to be an important facilitator of adopting healthy foods: 'Chinese people are cooking masters. We can make normal food special, even brown rice. It can be cooked in so many ways by Chinese' (focus group, site B, male physicians).

Table 1 Characteristics of participants in each focus group

\begin{tabular}{|c|c|c|c|c|c|c|c|}
\hline & $\mathbf{n}$ & Sex & Age (years) & BMI $\left(\mathrm{kg} / \mathrm{m}^{2}\right)$ & Overweight $^{\star}$ & $\begin{array}{l}\text { Eat at canteen } \geq 3 \\
\text { times/week }\end{array}$ & $\begin{array}{l}\text { Individual annual income } \\
\geq 90000 \text { RMB ( US } \$ 13560 \text { ) }\end{array}$ \\
\hline \multicolumn{8}{|l|}{ Site A (urban) } \\
\hline $\begin{array}{l}\text { Physicians and other } \\
\text { healthcare personnel† }\end{array}$ & 10 & Female & $37.8(4.4)$ & $21.5(1.6)$ & $10 \%(1)$ & $70 \%(7)$ & $90 \%(9)$ \\
\hline $\begin{array}{l}\text { Physicians and other } \\
\text { healthcare personnel† }\end{array}$ & 10 & Male & $34.3(6.1)$ & $23.7(3.0)$ & $50 \%(5)$ & $100 \%(10)$ & $60 \%(6)$ \\
\hline Nurses & 8 & Female & $40.4(8.7)$ & $21.5(2.5)$ & $25 \%(2)$ & $75 \%(6)$ & $70 \%(7)$ \\
\hline $\begin{array}{l}\text { Physicians and other } \\
\text { healthcare personnel† }\end{array}$ & 8 & Female & $41.3(8.0)$ & $22.9(2.4)$ & $25 \%(2)$ & $13 \%(1)$ & $100 \%(8)$ \\
\hline $\begin{array}{l}\text { Physicians and other } \\
\text { healthcare personnel† }\end{array}$ & 9 & Male & $44.4(10.9)$ & $25.4(2.3)$ & $78 \%(7)$ & $67 \%(6)$ & $89 \%(8)$ \\
\hline
\end{tabular}

Values are mean (SD) or per cent (n).

${ }^{*}$ Overweight defined as $\mathrm{BMI} \geq 24 \mathrm{~kg} / \mathrm{m}^{2}$, according to the Chinese government guideline. ${ }^{8}$

†Other healthcare personnel were primarily pharmacists.

BMI, body mass index. 
However, what constituted 'eating well' in terms of taste and cooking methods varied across regions of origin. For example, a local employee remarked, 'Dr. [X] is from the South, so he may not like it [the canteen food]' (focus group, site B, female nurses), whereas a non-local employee thought, 'The local chefs use sugar almost in every dish, and very heavy oil too. If I eat at home, I only bring the vegetables to boil and eat it; but the Shanghai people don't like it without oil' (interview, site A, CHC administrator).

\section{Subtheme $1 \mathrm{~b}$ : balancing taste preferences and nutrition}

Many participants expressed that unhealthy food tastes better than healthy food, for example, 'A: I have studied for a dietitian [certificate] [laughs]. Our teacher told us during the class that when considering nutritional value, we don't consider the taste, and sometimes in the Chinese cuisine there will be a conflict, that is, if the taste is good, the calories go up. B: In fact, what is delicious is not healthy ... A: Yes, what tastes good can't be healthy. B: Unhealthy food tastes great. C: Junk foods are the best [laughs]' (focus group, site A, female physicians). This cultural perception of unhealthy foods unequivocally tasting better than healthy foods was also expressed by men, who felt that the healthfulness of foods was a barrier to eating well: 'Speaking of eating healthy, in fact, I think eating well is important as well. Because if you only eat from the health point of view, it's hard, hard to eat well' (focus group, site A, male physicians).

Demographic factors influenced the balance between taste preference and nutrition. Participants believed that elderly people tend to choose foods based on nutrition whereas younger people on taste: 'Maybe because I'm younger and I have no health problem, I would choose white rice as it tastes better. I might consider coarse grains when I get older and become less healthy' (focus group, site B, male physicians). Women also believed that they valued nutrition more so than men: 'There are times when I eat with my supervisor. When I say the dish is too oily, my supervisor would complain that vegetable doesn't taste good without oil [laughs] ... It is very obvious that the male colleagues have different preferences' (interview, site A, CHC administrator). Recognising that this balancing act varies across individuals, canteen staff cited this as one of the primary reasons for not improving the quality and variety of canteen food. For example, one canteen manager said, 'If I provide them with more choices, I don't know what they'd like. I don't know if they'd want healthier or tastier food. Everyone's choice is different. There are people who prefer taste over health, also people who prefer health to taste' (interview, site A, canteen manager).

\section{Subtheme 1c: importance of food safety in healthfulness}

Food safety was considered a prerequisite for healthfulness by participants. In Chinese culture, 'food safety' encompasses everything from unintentionally contaminated foods (consistent with Western definitions of 'food safety') to intentional processing and/or illegal adulteration of foods (eg, foods with chemical additives and GMO foods are often considered 'unsafe'). Employees prefer to eat at the canteen because they perceive outside food to be unsafe, whereas they trust the canteen food quality relative to outside food. For example, one participant remarked: 'A: Recently our canteen is under renovation, so I have to go outside to eat. I suddenly found that many of the foods outside are not good either, because you know, the restaurants outside are ... like ...B: [you don't know if] the material they used were healthy or not. A: So I feel that our canteen is better, that I can trust it more, so I feel like returning to the canteen again. It's a dilemma' (focus group, site A, female physicians). A CHC administrator agreed: 'The raw food they [the canteen] purchase must be healthy. We trust them on that; including the condiments. They won't use unhealthy seasoning or flavoring just to boost the appearance of the dish. I think this is why they are healthier than the restaurants.'

This was especially true with respect to oil as several focus group participants and in-depth interviewees explicitly mentioned concerns about oil quality at restaurants, for example: 'The canteen would not use waste oil, I believe. But if we dine out, it's hard to tell whether the restaurants would use safe oil. So the most important thing to me is food safety' (focus group, site B, male physicians). However, there were some exceptions to this: a couple of focus group participants questioned the quality of the canteen food, for example, 'For some dishes [ground pork with yellow sauce], there is a more than $80 \%$ dislike rate [laughs] ... we don't know the quality of ground pork' (focus group, site A, male physicians) and, '... I can't accept the canteen's sanitary condition and taste. So I don't go to the canteen' (focus group, site B, female physicians).

\section{Theme 2: balancing acts that influence organisational decisions about food offerings}

Subtheme 2a: balancing canteen budget and food safety with the variety and quality of food offerings

Managers at both CHCs identified limited canteen budget as the primary barrier for improving food offerings: 'I hope that the canteen could be even better than general households, in terms of healthy eating, since we are a professional institution anyways. The barriers now are more on the cost side, not the awareness, right? ... This [cost] is the biggest barrier' (interview, site B, CHC administrator).

Employees felt that the canteen was good value. However, this value came at the cost of making improvements to the canteen in terms of increasing the variety of options available: 'The price and value of the food here are pretty good. It's very affordable, although the options are too few' (focus group, site B, male physicians). This was important because a lack of variety in food offerings was recognised as a significant source of dissatisfaction among employees. For some, variety was recognised as being more important than taste, for example, 'No 


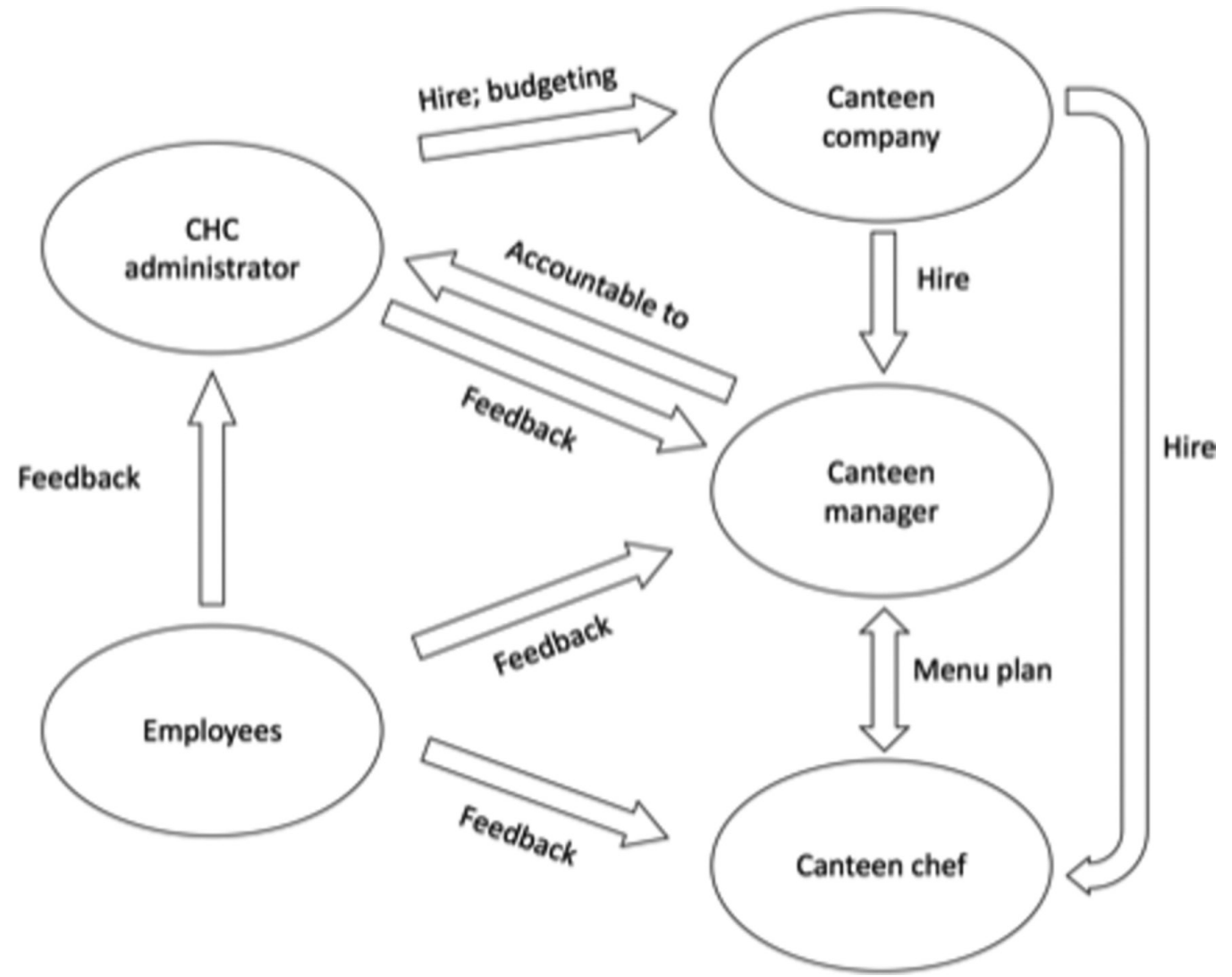

Figure 1 Interactions between key stakeholders in the worksite food environment as identified in interviews and focus groups discussions. $\mathrm{CHC}$, community health centres.

matter how delicious a dish is, if every day we eat the same thing, it's not delicious anymore' (focus group, site A, male physicians). In some cases, the lack of variety led employees to order food from outside or bring food from home, for example, 'A: There are too few choices at the canteen. You will sooner or later get bored, right? Certainly you will eat outside for diversity. B: Correct. The same dishes come back every week [laughs]' (focus group, site A, male physicians), and, 'We all ate at the canteen when we first time came here. As time went on, subconsciously, we don't have any interest in the canteen anymore, so we bring our own food' (focus group, site B, female physicians). There were exceptions to this, where employees were content with the lack of variety because, for example, they only ate one meal at the canteen: 'I usually have nicer food for dinner, so other things don't matter' (focus group, site B, female nurses).

In particular, participants desired an increase in the variety of cooking method. For example, 'It's fine to use the same food from week to week, but the flavor should keep changing' (interview, site A, CHC administrator), and, 'we would like to have more cooking methods, a little more of steaming or boiling dishes' (interview, site A, female physicians). Frying was perceived as an unhealthy alternative to steaming, boiling and braising. For example, although fish was typically served as a deepfried dish, many participants voiced their preference for steamed fish. However, one major barrier to freshness was cost: 'We can make steamed fish, but people won't eat it. We are not able to buy fresh fish in the morning, so we buy frozen fish. Steamed frozen fish has that fishy smell that people don't like ... We can only afford frozen fish' (interview, site A, canteen manager). Overall, employees expressed their desires for fresher foods and admitted that if raw ingredients are fresh there would be no need for much processing or seasoning. Vegetables in the canteen of site B, sourced directly from a local farm, were praised by a $\mathrm{CHC}$ administrator: 'The vegetables here are very fresh ... The vegetables from the city are not so fresh. If you go to the field here, you can have as much lettuce as you want in the winter." However, freshness was of less importance if the vegetables were not prepared properly; several focus groups in both sites raised the issue that vegetables were often ' overcooked'.

Food safety also factored into this balancing act. Incidentally, the two canteens we sampled are only licensed to serve foods with a minimum serving temperature, limiting the variety of cooking methods that can be used in the canteens. Canteen managers at both sites remarked: 'The core temperature [of served food items] has to be above $70^{\circ} \mathrm{C}$...You need to have a specific room for cold dishes. We don't have that' (interview, site B, canteen manager), and 'You see, in fact the cold dishes are healthy dishes ... In the restaurants outside, lettuces can be served as cold dish. But I can't. I have to use oil to stir-fry' (interview, site A, canteen manager). This restriction was reflected 
by employees: one focus group member was hesitant for the chef to cut watermelons because of concerns for lack of cleanness (focus group, site A, female physicians). Although serving whole fruits was not prohibited according to safety regulations, one manager was hesitant: 'Some sellers use special chemicals to preserve the fruits, which might cause harm. I don't want to risk that. There's a regulation saying that if more than 5 people get sick after a meal, the blame is on us. I can only make sure the preparation process here is safe, but I can't guarantee anything other than that' (interview, site A, canteen manager). The need to cater for large numbers also influenced the variety of feasible cooking methods, for example, 'Because the canteen cooks with a huge pot, which does not allow sophisticated food processing ... So even though we have a lot of recipes in hand, there is not much to choose from' (focus group, site A, male physicians).

\section{Subtheme $2 \mathrm{~b}$ : personnel, communication and management}

Interactions between employees, CHC administrators, canteen chefs and canteen managers (figure 1) also shape the worksite food environment. One CHC administrator felt the canteen improved a lot in the past twoyears ... after some communications' (interview, site A, CHC administrator), while another praised the canteen company because of its responsiveness: 'Whatever suggestions and feedback we have, they will try their best to adjust' (interview, site B, CHC administrator). In direct contrast to this, employees felt that the canteen staff were not responsive to their feedback, commenting: 'Basically if we told them something, they will change for a short period of time. But then returned [to what it used to be]' (focus group, site A, female nurses). The chef's habits were widely quoted as an obstacle to reducing the amount of oil and salt at one of the sites: '[the chef's] cooking method is heavy oil and heavy salt, there's no use to tell them, it's the same old thing' (focus group, site A, female nurses), and, '[the chef] has all the spices mixed together. So with a spoonful of seasonings, there is no way to tell him to put a little less salt' (focus group, site A, female physicians). Furthermore, the canteen chef and manager may not be incentivised to respond to individual employees' suggestions if these needs involve structural changes. The canteen was primarily accountable to the CHC administrators, who were responsible for hiring the canteen company. The $\mathrm{CHC}$ administrators, on the other hand, would only act to improve the canteen based on feedback from the employees.

All stakeholders perceived a lack of communication regarding canteen offerings. An administrator disclosed to the interviewer: 'I wish that the employees could bring [potential canteen interventions] up. If employees made the suggestions, and the majority think that's a legit suggestion, then it can happen' (interview, site A, CHC administrator). A canteen manager agreed: 'Maybe they [the employees] don't feel comfortable talking to me

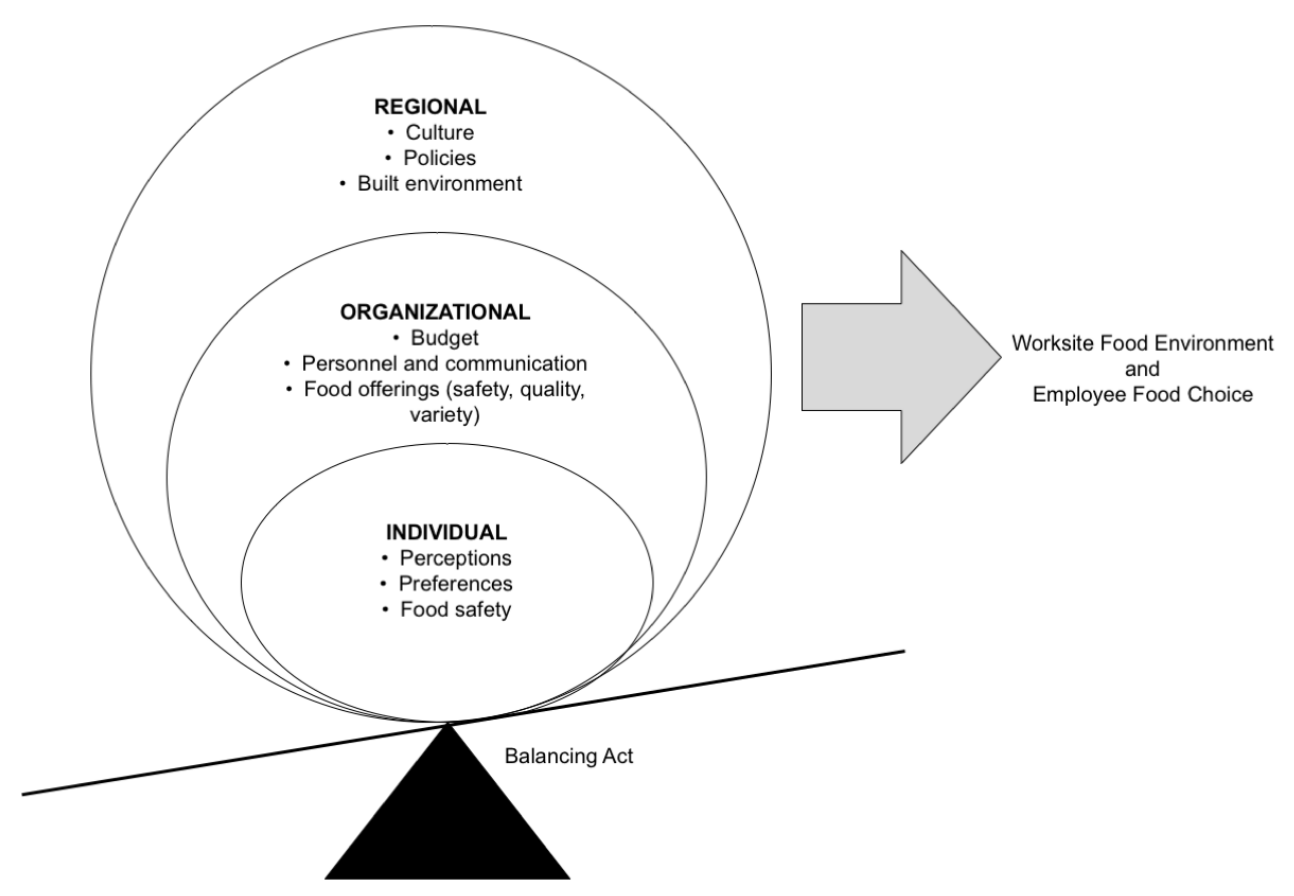

Figure 2 Conceptual framework capturing balancing acts at the individual level (cultural perception of 'eating well', balancing taste preferences and nutrition, importance of food safety in healthfulness) and organisation level (balancing canteen budget and food safety with the variety and quality of food offerings, personnel, communication and management). 
directly; maybe they discuss our work but don't let us know' (interview, site A, canteen manager). Employees also expressed concern over the lack of communication. For example, they complained, 'I don't even know where the oil [in the canteen soup] comes from' (focus group, site A, male physicians) and 'they've [canteen staff] never communicated with us' (focus group, site B, female physicians).

\section{Conceptual framework}

The two themes and five subthemes informed the development of the conceptual framework (figure 2), which is based on the ecological model. ${ }^{9}$ Individual-level factors relate to employees' cultural perceptions of 'eating well', taste preferences and perceptions of the importance of food safety in healthfulness, all of which impact food choices. Organisation-level factors relate to resource availability, constraints in offerings due to safety regulations and the interplay between different key players. These factors all influence the social and physical environments of CHC canteens, and in particular, the food offerings. Underlying regional and national drivers emerged when delving deeper into the subthemes, including the cultural importance of Chinese cooking and varieties in regional culinary traditions, the overarching impact of regulations, policies and nutrition promotions, and the limitations imposed by national food safety concerns. Balancing acts at each level shape employee food choice and worksite food environment. The theoretical framework informed the selection of specific interventions that are likely to be effective in this specific context (table 2).

Table 2 Overview of theory-based methods and practical strategies to be used in community health centre (CHC) canteenbased intervention

\begin{tabular}{ll}
\hline $\begin{array}{l}\text { Level of the } \\
\text { intervention }\end{array}$ & $\begin{array}{l}\text { Theory-based } \\
\text { methods }\end{array}$ \\
\hline
\end{tabular}

Individual Education

\section{Facilitation}

Media advocacy Coalition forming

\section{Practical strategies informed by focus groups}

Many employees expressed the sentiment that as medical professionals, 'we know what we should know' (focus group, site A, female physicians). Further interventions targeting nutrition knowledge on effects of specific nutrients (eg, oil and salt) may not yield much effect.

However, when asked about whether weight control was important, one focus group member remarked: 'No one here needs to lose weight; we need to gain weight' (focus group, site A, male physicians). Interestingly, for this group of male physicians, $50 \%$ were overweight, indicating a lack of awareness for risk of overweight. Additional information on the health effects of genetically modified foods and on added sugars may also be helpful in shaping food choices.

Dietitian involved in menu planning. For example: 'Things like calories, food composition, it's impossible for me to look into these details. I'm not specialized in canteen management, nor am I a nutritionist; I wasn't trained in this way' (interview, site A, canteen manager). And: a dietitian 'will supervise the use of oil, salt, and sugar' (focus group, site A, male physicians), 'enrich the variety' (focus group, site A, female nurses) and 'design a nutritional meal plan' that employees 'can even learn from' (focus group, site A, female physicians).

Skilled chef involved in menu planning. For example: at site $\mathrm{B}$, a chef with a level 2 culinary certificate cooks at the canteen. Both the $\mathrm{CHC}$ and canteen managers recognised that cooking skill of the chef was a main determinant for employee satisfaction, and in all focus groups, employees expressed their satisfaction with the taste of canteen food.

Increase subsidy for specific items, as has been done previously for nongenetically modified oil at site A. Target fruits, yoghurts and whole grains. For example: 'Instead of increase the budget of 1 CNY to improve the dish, we would rather choose to have that for fruits or yogurts. If the budget goes directly to certain food, it's more acceptable' (interview, site A, CHC manager).

Advertising changes to menu in terms of 'eating well' not ' healthfulness'.

Create organisation-level coalition of canteen manager, $\mathrm{CHC}$ administrator and employee representatives to collect feedback on specific menus, leveraging social media and mobile technology. For example, employees argued that waste can be minimised if people's preferences for specific choices could be predetermined: 'If they have more type of dishes. I don't mind of getting less quantity. It won't be a waste like this. They should ask what people like roughly' (focus group, site B, female physicians). 


\section{DISCUSSION}

This article describes the formative research process whereby we identified determinants of the food environment and employee food choice at two CHCs in Shanghai, China. Three subthemes were identified as important for influencing individual food choice: the cultural perception of 'eating well', the need to balance taste preferences and nutrition, and the emphasis on food safety in healthfulness. At the organisation level, two related subthemes emerged: the balance of canteen budget and food safety with the variety and quality of offerings, and the interplay between key stakeholders. There is a very limited literature on determinants of food provision and consumption in the workplace, and those studies that have been published were largely conducted in the USA and Europe. ${ }^{1011}$ This is the first qualitative study to explore determinants of food provision and consumption in Chinese worksites, and thus our findings will prove to be important for planning interventions in the unique and complex food environment of worksites.

We confirmed the value of 'eating well' in Chinese culture in driving employee food choice ${ }^{12}$ : an intervention that emphasises traditional local foods and cooking styles is likely to be more acceptable to participants than one emphasising healthy foods from other cultures. For example, while salad bars may be installed at worksite canteens in Western countries to increase vegetable intake, such an intervention is unlikely to be effective in China where raw vegetables are not traditionally desired as standard lunch dishes. What constituted 'eating well' was influenced by deeply engrained cultural ideals and personal preferences-and thus could prove to be an important barrier to changing diets. However, it is encouraging that we found at the site with an experienced and trained chef that many employees commended him on his braised dishes in terms of taste and healthfulness, suggesting that even with local cuisine the use of salt and oil could be reduced while maintaining taste.

Consistent with findings from two previous qualitative studies in the UK, one conducted among non-academic employees of a university, ${ }^{13}$ the other among public sector employees, ${ }^{10}$ we found that nutrition did not take precedence when making decisions about food at canteens. Among our study participants, all of whom were trained healthcare providers, heavy use of oil and salt was universally recognised as unhealthy, but taste was prioritised over healthfulness. This suggests that a generic nutrition education intervention is unlikely to produce significant behaviour changes in this setting, and indeed, previous worksite interventions focused on educational campaigns (eg, nutrition presentations, posters in canteens, distribution of materials to employees) have shown limited improvements in dietary intake. ${ }^{1415}$ Personalised nutrition messaging may produce greater improvements in dietary intake. ${ }^{16}$ In our setting, such messaging could prove especially useful among men, some of whom expressed discordance between their own health status (overweight) and the need for dietary improvements. A web-based survey of physicians in the USA found that physicians' healthy lifestyle habits are correlated with higher likelihood of lifestyle modification counselling for hypertensive patients. ${ }^{17}$ This has important implications for future interventions aimed at improving not only employee health at CHCs in China, but also, potentially, the provision of healthy lifestyle advice to patients who are overweight or obese.

At the organisation level, there is a balancing act between restraining factors (budget and food safety regulations) and desired outcomes (food variety and quality). As has been documented in previous qualitative research in the $\mathrm{UK}^{10}$ and the Netherlands, ${ }^{18}$ the company providing food to the worksite canteens must run a financially viable service, which means that their priority is providing food that employees will buy-not necessarily food that will promote employee health. When marketing the new canteen menus, a practical strategy for overcoming this organisation-level barrier may be the adoption of a media campaign that leverages the perceived importance of 'eating well' rather than 'healthfulness'. This innovative approach to food marketing at worksites should be evaluated considering the limited and inconsistent evidence testing social media campaigns in terms of improving dietary intake. ${ }^{19}$ In addition, as was brought up by participants of this study, improving communication between $\mathrm{CHC}$ administrators, canteen managers and staff, and employees may address this barrier. Consistent with Kotter's eight-stage process, ${ }^{20}$ creating an organisation-level coalition of these stakeholders to collect feedback on specific menus by leveraging social media and mobile technology could achieve change in the worksite food environment.

We found that although food safety is important both at individual and organisational levels, well-intentioned safety regulations ${ }^{21}$ for worksite canteens-including the emphasis on core serving temperature and the need for a specific raw-dish preparation room-limited cooking methods, restricted variety of food offerings and diminished taste and presentation. The optimal solution would be to establish a raw-dish preparation room and acquire appropriate licences to serve cold dishes. In the absence of additional space or funds for infrastructure, employing a skilled chef and dietitian is likely necessary to improve the healthfulness and variety of canteen food. Increasing the CHC budget for specific items such as raw fruits and yoghurt may also prove effective.

Similar to the aforementioned qualitative study among public sector workers in the UK, ${ }^{10}$ we did not find strong evidence to support that providing nutrition information influences food choice. One randomised-controlled trial in Germany ${ }^{22}$ found that although nutrition information given in the form of food labels has an impact on the perceived healthfulness of foods, this perceived healthfulness is unlikely to influence food choice and food intake. Similarly, a canteen intervention at Harvard University that included traffic light labelling, choice architecture and 'healthy plate' tray stickers failed to demonstrate any improvements in the healthfulness of purchases. ${ }^{23}$ Our 
interview guide explicitly queried focus group participants about their perceptions regarding the usefulness of traffic light labelling systems, and participants said the local government had already implemented such programmes with no effect. While individuals may want nutritional information, especially calories ${ }^{13}$ available to them, environmental changes such as changing the healthfulness of food offerings are likely to be necessary for exacting change in eating behaviours.

There are several strengths of our study. We conducted five in-depth interviews and six focus groups: this relatively large sample represents healthcare workers from both rural and urban CHCs in Pudong. The willingness to discuss food in the Chinese culture promoted a high level of engagements of both the employees and the CHC directors, which allowed us to identify multitudes of enablers, barriers and potential interventions driven by employees. The interviewer (RL), a native speaker who understands local culture, coded the transcripts and analysed emerging themes, ensuring minimal discrepancy between field observations and subsequent analyses. Furthermore, many participants perceived that a collaboration with a local government agency and a Western institution could bring additional resources to improving canteen food service, so were open to discussing their opinions on an ideal food environment at their worksite.

There are also several limitations of our study worth noting. The interviewer was a trainee from a US institution; this may have led to bias in the focus group discussions with a greater emphasis on the value of traditional Chinese cooking in contrast to Western cooking. However, the interview guide was dynamic and updated in response to these concerns early on, enabling us to improve the richness of subsequent data. Moreover, employees who volunteered to be in the focus groups may be more interested in healthy food and supportive of making changes to the canteen. Finally, at the time of our visit, there were few opportunities for us to directly observe 'usual' canteen food because the canteens were under construction or recent restructuring at both sites.

Essential elements for a successful community intervention programme include a good understanding of the community ('community diagnosis') and full participation of the people themselves. ${ }^{24}$ The intervention programme developed from this study will directly incorporate the formative research findings presented here and will be grounded in the ecological approach. The intervention will target both organisational and individual factors, and aim to reduce barriers identified and enable the facilitators. Local and cultural adaptation will be necessary to enhance deliverability in all participating CHCs and increase long-term programme sustainability.

Acknowledgements The authors thank colleagues at the Pudong Institute for Health Development for their support with this study, Zhiqun Shu for the loan of the recorder and help with transportation, administrators in the sampled community health centers for logistical support and their commitment to improving employee well-being and public health, and all those who volunteered to be interviewed.
Contributors RL, LJ and LMJ conceived the research question and contributed to the study design. LJ identified study sites and coordinated local support for data collection. RL collected the data and coordinated transcription. YW and RL translated the transcripts. RL and LMJ analysed the data. RL and YW drafted the initial manuscript with support from LMJ. All authors provided substantive feedback on the manuscript and approved the final version.

Funding Funding was provided by the Harvard T.H. Chan School of Public Health. Competing interests None declared.

Patient consent Obtained.

Ethics approval This study was approved by the Institutional Review Board of Harvard University (ref no: IRB16-1424).

Provenance and peer review Not commissioned; externally peer reviewed.

Data sharing statement The dataset analysed during the current study is available from the corresponding author on reasonable request.

Open Access This is an Open Access article distributed in accordance with the Creative Commons Attribution Non Commercial (CC BY-NC 4.0) license, which permits others to distribute, remix, adapt, build upon this work non-commercially, and license their derivative works on different terms, provided the original work is properly cited and the use is non-commercial. See: http://creativecommons.org/ licenses/by-nc/4.0/

(C) Article author(s) (or their employer(s) unless otherwise stated in the text of the article) 2018. All rights reserved. No commercial use is permitted unless otherwise expressly granted.

\section{REFERENCES}

1. Adair LS, Gordon-Larsen P, Du SF, et al. The emergence of cardiometabolic disease risk in Chinese children and adults: consequences of changes in diet, physical activity and obesity. Obes Rev 2014;15:49-59.

2. Lu J, Bi Y, Ning G. Curbing the obesity epidemic in China. Lancet Diabetes Endocrinol 2016;4:470-1.

3. World Bank Group. World Development Report 2013 on Jobs Statistical Tables. http://data.worldbank.org/data-catalog/wdr-2013jobs (accessed 23 Oct 2017).

4. Allan J, Querstret D, Banas K, et al. Environmental interventions for altering eating behaviours of employees in the workplace: a systematic review. Obes Rev 2017;18:214-26.

5. Li H, Liu K, Gu J, et al. The development and impact of primary health care in China from 1949 to 2015: A focused review. Int $J$ Health Plann Manage 2017;32:339-50.

6. Department of Planning and Information, National Health and Family Planning Commission. Statistical Communique on the Development of Health and Family Planning in China in 2016. http://www.nhfpc .gov.cn/guihuaxxs/s10748/201708/d82fa7141696407abb4e f764f3edf095.shtml (accessed 23 Oct 2017).

7. Helfand BK, Mukamal KJ. Healthcare and lifestyle practices of healthcare workers: do healthcare workers practice what they preach? JAMA Intern Med 2013;173:242-4.

8. National Health and Family Planning Commission. Criteria of Weight for Adults. 2013 http://www.nhfpc.gov.cn/ewebeditor/uploadfile/ 2013/08/20130808135715967.pdf (accessed 23 Oct 2017).

9. Bowen DJ, Barrington WE, Beresford SA. Identifying the effects of environmental and policy change interventions on healthy eating. Annu Rev Public Health 2015;36:289-306.

10. Pridgeon A, Whitehead K. A qualitative study to investigate the drivers and barriers to healthy eating in two public sector workplaces. J Hum Nutr Diet 2013;26:85-95.

11. Mazzola JJ, Moore JT, Alexander K. Is work keeping us from acting healthy? How workplace barriers and facilitators impact nutrition and exercise behaviors. Stress Health 2017;33:479-89.

12. Swislocki M. Culinary nostalgia regional food culture and the urban experience in Shanghai. Palo Alto, CA: Stanford University Press, 2009.

13. Thomas EL, Puig Ribera A, Senye-Mir A, et al. Promoting Healthy Choices in Workplace Cafeterias: A Qualitative Study. J Nutr Educ Behav 2016;48:138-45.

14. Sorensen G, Stoddard A, Peterson K, et al. Increasing fruit and vegetable consumption through worksites and families in the treatwell 5-a-day study. Am J Public Health 1999;89:54-60.

15. Bandoni DH, Sarno F, Jaime PC. Impact of an intervention on the availability and consumption of fruits and vegetables in the workplace. Public Health Nutr 2011;14:975-81. 
16. Celis-Morales C, Livingstone KM, Marsaux CF, et al. Effect of personalized nutrition on health-related behaviour change: evidence from the Food4Me European randomized controlled trial. Int $J$ Epidemiol 2017;46:578-88.

17. Hung OY, Keenan NL, Fang J. Physicians' health habits are associated with lifestyle counseling for hypertensive patients. Am J Hypertens 2013;26:201-8.

18. Steenhuis I, van Assema P, Reubsaet A, et al. Process evaluation of two environmental nutrition programmes and an educational nutrition programme conducted at supermarkets and worksite cafeterias in the Netherlands. J Hum Nutr Diet 2004;17:107-15.

19. Williams G, Hamm MP, Shulhan J, et al. Social media interventions for diet and exercise behaviours: a systematic review and meta-analysis of randomised controlled trials. BMJ Open 2014;4:e003926.
20. Kotter JP. Leading Change. Brighton Watertown, MA: Harvard Business Review Press, 1996.

21. Shanghai Municipal Food and Drug Administration. Shanghai Municipal Food Business License Management Practices (Trial version). http://www.shfda.gov.cn/gb/node2/yjj/xxgk/zfxxgk/zxxxgk/ sp/u1ai51027.html (accessed 23 Oct 2017).

22. Borgmeier I, Westenhoefer J. Impact of different food label formats on healthiness evaluation and food choice of consumers: a randomized-controlled study. BMC Public Health 2009;9:184.

23. Seward MW, Block JP, Chatterjee A. A Traffic-Light Label Intervention and Dietary Choices in College Cafeterias. Am J Public Health 2016;106:1808-14.

24. World Health Organization. Global Action Plan for the Prevention and Control of Noncommunicable Diseases 2013-2020. Geneva: World Health Organization, 2013. 